\title{
Comportamentos de risco de pacientes oncológicos em follow up
}

Risk behaviors of oncological patients in follow up

Conductas de riesgo de los pacientes oncológicos en seguimento

Natália Vinhando ${ }^{1}$ (D) https://orcid.org/0000-0001-5379-3276

Débora Rebollo de Campos $^{1}$ (D) https://orcid.org/0000-0002-7038-2345

\section{Resumo}

Objetivo: Identificar comportamentos de risco de pacientes em follow up que realizaram tratamento oncológico. Método: Estudo transversal e descritivo realizado no Hospital de Câncer de Barretos no período de 20/12/2018 à 28/08/2019 com pacientes sobreviventes do câncer infantojuvenil.

Resultados: Foram entrevistados 71 pacientes (52\% sexo feminino) e a idade destes variou de 16 a 39 anos. 0 tempo médio fora de terapia foi de 10 anos. Mais de $50 \%$ estavam trabalhando. Sobrepeso foi observado em $37,5 \%$ dos participantes entre 16 e 19 anos e em 25\% com mais de 19 anos. Cerca de $38 \%$ experimentaram tabaco e $84,5 \%$ bebida alcoólica. As drogas ilícitas foram utilizadas ao menos uma vez por $20 \%$ dos pacientes. A faixa etária média de início da atividade sexual foi 17 anos.

Conclusão: Os dados obtidos nesta pesquisa podem proporcionar uma visão ampliada dos aspectos sociais demandados pelos pacientes após anos de término de terapia e auxiliar toda a equipe na prestação de cuidados para o reestabelecimento da vida desses indivíduos.

\section{Abstract}

Objective: To identify risk behaviors of patients in follow-up who underwent cancer treatment.

Method: Cross-sectional and descriptive study conducted at the Hospital de Câncer de Barretos from 12/20/2018 to $8 / 28 / 2019$ with survivors of childhood cancer.

Results: 71 patients were interviewed (52\% female) and their ages ranged from 16 to 39 years. Mean time out of therapy was 10 years. More than $50 \%$ were working. Overweight was observed in $37.5 \%$ of participants between 16 and 19 years old and in $25 \%$ over 19 years old. About $38 \%$ had tried tobacco and $84.5 \%$ had tried alcohol. Illicit drugs were used at least once by $20 \%$ of patients. The average age at which sexual activity began was 17 years. Conclusion: The data obtained in this research can provide an expanded view of the social aspects required by patients after years of therapy completion and help the entire team in providing care for the restoration of these individuals' lives.

\section{Resumen}

Objetivo: Identificar conductas de riesgo en pacientes en seguimiento sometidos a tratamiento oncológico.

Método: Estudio transversal y descriptivo realizado en el Hospital de Câncer de Barretos del 20/12/2018 al 28/08/2019 con supervivientes de cáncer infantil.

Resultados: Se entrevistaron 71 pacientes (52\% mujeres) y sus edades oscilaron entre los 16 y los 39 años. Aproximadamente el $80 \%$ eran de la región sureste. El tiempo medio fuera de la terapia fue de 10 años. Más del $50 \%$ estaban trabajando. Se observó sobrepeso en el 37,5\% de los participantes entre 16 y 19 años y en el $25 \%$ mayores de 19 años. Aproximadamente el 38\% había probado el tabaco y el 84,5\% había probado el alcohol. El $20 \%$ de los pacientes consumió drogas ilícitas al menos una vez. La edad promedio de inicio de la actividad sexual fue de 17 años.

Conclusión: Los datos obtenidos en esta investigación pueden proporcionar una visión ampliada de los aspectos sociales requeridos por los pacientes después de años de finalización de la terapia y ayudar a todo el equipo a brindar atención para la restauración de la vida de estas personas.

\section{Descritores}

Saúde do adolescente; Neoplasias; Sobreviventes de câncer; Enfermagem pediátrica

\section{Keywords}

Adolescent health; Neoplasms; Cancer survivors; Pediatric nursing

\section{Descriptores}

Salud del adolescente; Neoplasias; Supervivientes de cáncer; Enfermería pediátrica

\section{Como citar:}

Vinhando N, Campos DR. Comportamentos de risco de pacientes oncológicos em follow up. Rev Soc Bras Enferm Ped. 2021;21(2):159-65.

\footnotetext{
${ }^{1}$ Hospital de Câncer de Barretos, Barretos, SP, Brasil.

Conflitos de interesse: nada a declarar.

Submetido: 2 de Dezembro de 2021 | Aceito: 15 de Dezembro de 2021

Autor correspondente: Natália Vinhando | E-mail: navinhando@outlook.com

DOI: http://dx.doi.org/10.31508/1676-379320210022
} 


\section{Introdução}

O câncer que acomete crianças e adolescentes (de 0 a 19 anos) é considerado raro, quando comparado aos tumores que se desenvolvem em adultos, correspondendo de 1 a $4 \%$ de todas as neoplasias malignas, na maioria das populações. ${ }^{(1)}$

A leucemia é o tipo de câncer mais predominante na população infantil, correspondendo a $28 \%$ dos casos, seguida pelos tumores do sistema nervoso central $(26 \%)$ e os linfomas $(8 \%))^{(2)}$

Apesar dos estudos sugerirem a existência de potenciais fatores de risco para o câncer pediátrico, não há evidências científicas de que a doença possa ser prevenível. Logo, a prevenção ainda é um desafio para o futuro e a ênfase atual da abordagem a esse público está pautada na detecção precoce e no encaminhamento para um tratamento oportuno e de qualidade, que possibilite maiores chances de cura. ${ }^{(3)}$

Nos últimos anos, o progresso no tratamento do câncer infantojuvenil aumentou significativamente devido, em grande parte, as novas modalidades terapêuticas disponíveis, como o Transplante de Células Tronco Hematopoiéticas (TCTH), a imunoterapia e a terapia-alvo. ${ }^{(4)}$

Atualmente, a sobrevida para a população oncológica pediátrica, em países desenvolvidos, é em torno de $80 \%$, podendo variar conforme o tipo de câncer, a idade do paciente e outras características específicas. ${ }^{(5)}$ No Brasil, essa taxa fica em torno de $64 \%$ e em Barretos, a sobrevida global no período de 2000 a 2017 foi de 55,3\%, excluindo os Tumores Raros, as Síndromes Mielodisplásicas (SMD) e os Sarcomas não Rabdomiossarcomas. ${ }^{(6,7)}$

Nos estudos sobre os sobreviventes do câncer, não existe um consenso sobre quando inicia o follow up, período considerado de sobrevivência do paciente que realizou tratamento oncológico; se é após o tratamento, após a fase de cinco anos de diagnóstico de câncer ou o tempo de separação dos cuidados de fim de vida. ${ }^{(8)}$

As neoplasias malignas e o seu tratamento predispõem os jovens sobreviventes a uma variedade de efeitos tardios, sejam eles físicos e/ou psíquicos e/ ou sociais, que podem provocar altas taxas de morbimortalidade para essa população. Essas taxas podem ser potencializadas pelo envolvimento dos jovens so- breviventes em comportamentos típicos da idade, incluindo o tabagismo, uso de álcool e drogas ilícitas, obesidade e sexo desprotegido. ${ }^{(9)}$

Sobreviventes de leucemia linfoide aguda e tumores do sistema nervoso central estão mais propensos ao desenvolvimento de obesidade devido às modalidades terapêuticas empregadas, entretanto, não podem ser descartados os demais fatores contribuintes para o ganho de peso como má nutrição e sedentarismo, presentes também na população em geral. ${ }^{(10)}$

Taxas de tabagismo em sobreviventes permanecem maiores quando comparado ao grupo controle, fato que pode estar relacionado ao não direcionamento de ações preventivas a este público. ${ }^{(11)}$

A população crescente de indivíduos que sobreviveram ao câncer cria uma série de desafios aos profissionais de saúde. Compreender o impacto a longo prazo das terapias utilizadas é fundamental para melhorar o atendimento dos sobreviventes de câncer infantil na idade adulta. ${ }^{(5)}$

Os estudos populacionais apresentam os dados sobre comportamentos de risco de forma geral, não estratificando os pacientes oncológicos. Deste modo, o estudo visa entender, especificamente, como se encontram os pacientes em acompanhamento após término do tratamento oncológico, com relação aos comportamentos de risco, e ter uma melhor compreensão das peculiaridades e demandas destes sobreviventes, portanto, a pesquisa teve por objetivo identificar comportamentos de risco de pacientes em follow up que realizaram tratamento oncológico.

\section{Métodos}

Estudo transversal e descritivo realizado no ambulatório do Hospital de Câncer Infantojuvenil de Barretos, que está situado na região norte do estado de São Paulo e recebe, aproximadamente, 500 novos casos sugestivos de tumores ao ano, sendo que destes, são confirmados a malignidade de, aproximadamente, 300.

A amostra foi definida por conveniência baseando-se no número médio de pacientes que comparecem mensalmente às consultas de follow up nesta instituição. Os critérios de inclusão foram: pacientes que terminaram o tratamento oncológico há cinco anos ou mais; idade igual ou superior a 16 anos e; que 
compreendiam a língua portuguesa e conseguiam se comunicar com o pesquisador. Foram excluídos os pacientes que chegaram ao hospital com diagnóstico de câncer de outro serviço e iniciaram o tratamento oncológico na urgência, antes da revisão das lâminas da biópsia da origem ou nova biópsia, e porventura o diagnóstico era benigno.

A coleta de dados foi realizada por uma das pesquisadoras deste estudo (N.V) no período de 20/12/2018 à 28/08/2019 por meio de um questionário estruturado, baseado na Ficha de Coleta de Dados do Grupo de Estudos Pediátricos sobre Efeitos Tardios da Terapia Oncológica (GEPETTO) e a partir da consulta na literatura. As variáveis do estudo foram: identificação, dados sociodemográficos e comportamentos de risco (atividade sexual, obesidade e uso de tabaco, álcool e drogas ilícitas). Dados clínicos (diagnóstico, tratamento realizado, peso, altura e outros) foram obtidos a partir da consulta em prontuário do paciente.

$\mathrm{O}$ agendamento e a chegada do paciente ao serviço foram identificados através do sistema interno do hospital (SisOnco). Para participantes menores de 18 anos, a abordagem foi realizada na presença do responsável. Para os maiores de 18 anos, a presença do acompanhante ficou a critério do participante. Após esclarecimentos e aceite para participação na pesquisa, foi oferecido Termo de Assentimento e/ou Consentimento e o participante conduzido até uma sala reservada para coleta de dados. Se alguma das perguntas trouxesse lembranças desagradáveis, era oferecido atendimento com equipe da psicologia, se esta fosse a vontade do paciente ou acompanhante/responsável.

Os dados coletados foram armazenados na plataforma Research Electronic Data Capture (REDCap) e a amostra foi caracterizada a partir da média e desvio padrão para as variáveis quantitativas e pelas frequências absoluta e relativa, para as variáveis qualitativas. As análises foram realizadas a partir do software Statistical Package for the Social Sciences (SPSS) versão 21.

A pesquisa seguiu a resolução 466/2012 que versa sobre os aspectos éticos na pesquisa com seres humanos. O projeto de pesquisa foi submetido ao comitê de ética e pesquisa do Hospital de Câncer de Barretos e foi aprovado sob o número de protocolo 01482818.2.0000.5437. O estudo teve um risco mínimo para o participante da pesquisa (quebra de sigilo de forma acidental) e foram tomadas todas as medi- das necessárias para a preservação do anonimato dos participantes.

\section{Resultados}

Dos 71 pacientes que participaram da pesquisa, 52\% eram do sexo feminino. A faixa etária dos participantes variou de 16 a 39 anos, com média de idade de 24 anos. Quanto a cor de pele, $68 \%$ autodeclararam-se brancos e ao estado civil, $73 \%$ relataram ser solteiros. Em relação a região de procedência, $80 \%$ eram da região sudeste e quanto à religião, $45 \%$ eram católicos. Cerca de $10 \%$ dos entrevistados não possuem religião. Quanto à escolaridade, 35\% tinham o ensino superior incompleto, $31 \%$ o ensino médio completo e $18 \%$ o ensino superior completo. Os participantes residiam frequentemente com as mães (55\%), tinham entre dois e quatro salários mínimos (48\%) de renda familiar e dividiam essa renda com cerca de três $(27 \%)$ a quatro (27\%) pessoas. A tabela 1 mostra que $51 \%$ dos pacientes estavam trabalhando e destes, $44,4 \%$ tinham como vínculo empregatício o registro em carteira de trabalho. $\mathrm{O}$ fato de terem se submetido a um tratamento oncológico no passado foi relatado por $17 \%$ dos pacientes como algo que dificultou a contratação em um emprego.

Tabela 1. Distribuição dos dados sobre trabalho de uma amostra de pacientes em follow up tratados em um hospital oncológico do interior paulista

\begin{tabular}{lc}
\hline Variáveis & $\mathrm{n}(\%)$ \\
\hline Trabalham atualmente & $36(51)$ \\
Sim & $35(49)$ \\
Não & \\
Vínculo empregatício & $12(33,3)$ \\
$\quad$ Sem registro em carteira & $16(44,4)$ \\
Com registro em carteira & $8(22,2)$ \\
$\quad$ Autônomo & \\
Se sente ou sentiu dificuldade de encontrar emprego & \\
porque foi um paciente oncológico & $12(17)$ \\
Sim & $59(83)$ \\
$\quad$ Não & \\
\hline
\end{tabular}

Osteossarcoma foi o diagnóstico mais frequente com $27 \%$, seguido por Linfoma de Hodgkin com $11 \%$ e Linfoma não Hodgkin com 8,5\%. Os tumores do Sistema Nervoso Central (SNC) representaram 8,5\% dos diagnósticos. Cerca de $73 \%$ dos pacientes não tiveram metástase ao diagnóstico. Quanto às modalidades de 
tratamento, quimioterapia e cirurgia $(45 \%)$ foram as terapias mais utilizadas. O tempo de tratamento foi maior que 12 meses para $41 \%$ dos pacientes (Tabela 2). A média de tempo fora de terapia foi de 10,9 anos com desvio padrão de 4,5 .

Tabela 2. Distribuição dos dados de diagnóstico e tratamento de uma amostra de pacientes em follow up tratados em um hospital oncológico do interior paulista

\begin{tabular}{lc}
\hline Variáveis & $\mathrm{n}(\%)$ \\
\hline Diagnóstico & \\
Osteossarcoma & $19(27)$ \\
Linfoma Hodgkin & $8(11)$ \\
Linfoma não Hodgkin & $6(8,5)$ \\
Tumores do Sistema Nervoso Central & $6(8,5)$ \\
Leucemia Linfoide Aguda & $5(7)$ \\
Tumor de Wilms & $5(7)$ \\
Sarcoma de Ewing & $4(6)$ \\
Tumor de Células Germinativas & $4(6)$ \\
Sarcoma não Rabdomiossarcoma & $3(4)$ \\
Retinoblastoma & $2(3)$ \\
Leucemia Mieloide Aguda & $1(1)$ \\
Rabdomiossarcoma & $1(1)$ \\
Outros* & $7(10)$ \\
Metástase ao diagnóstico & \\
Sim & $19(27)$ \\
Não & $52(73)$ \\
Modalidades de tratamento & \\
Quimioterapia e cirurgia & $32(45)$ \\
Quimioterapia e radioterapia & $14(20)$ \\
Quimioterapia & $9(13)$ \\
Quimioterapia, radioterapia e cirurgia & $8(11)$ \\
Cirurgia & $3(4)$ \\
Outros* & $5(7)$ \\
Tempo de tratamento & \\
1 a a meses & \\
4 meses & $5(7)$ \\
\hline
\end{tabular}

*Outros: Carcinoma de Nasofaringe, Carcinoma Papilífero de Tireóide, Cistoadenocarcinoma Mucinoso de Ovário, Tumor de Células da Granulosa em Ovário, Histiocitose de Células de Langerhans, Sarcoma de Células Claras Renais; ${ }^{* *}$ Outros: Iodoterapia e TCTH associado a quimioterapia e/ou radioterapia e/ou cirurgia para remoção do tumor

Com relação à menarca, $100 \%$ das pacientes já iniciaram o ciclo menstrual e dessas, $38 \%$ referiram ter o ciclo irregular. Do total de participantes, $87 \%$ já iniciaram as atividades sexuais e cerca de $45 \%$ não utilizam métodos contraceptivos (Tabela 3). A média de idade de início das atividades sexuais foi de 17 anos com desvio padrão de 3 . Cerca de $77,5 \%$ dos participantes nunca engravidaram e 22,5\% engravidaram por fertilização natural. Do percentual que tem filhos vivos (81\%), $62 \%$ tem um filho e $8 \%$ tem filhos adotados (Tabela 3).
Tabela 3. Distribuição dos dados de ciclo menstrual, sexualidade e gravidez de uma amostra de pacientes em follow up tratados em um hospital oncológico do interior paulista

\begin{tabular}{|c|c|}
\hline Variáveis & $\mathrm{n}(\%)$ \\
\hline \multicolumn{2}{|l|}{ Iniciaram o ciclo menstrual } \\
\hline Sim & $37(100)$ \\
\hline \multicolumn{2}{|c|}{ Regularidade do ciclo menstrual } \\
\hline Ciclo menstrual regular & 20(54) \\
\hline Ciclo menstrual irregular & $14(38)$ \\
\hline Outros* & $3(8)$ \\
\hline \multicolumn{2}{|l|}{ Início da atividade sexual } \\
\hline Sim & $62(87)$ \\
\hline Não & $9(13)$ \\
\hline \multicolumn{2}{|c|}{ Uso de método contraceptivo** } \\
\hline $\operatorname{Sim}$ & $39(55)$ \\
\hline Não & $32(45)$ \\
\hline \multicolumn{2}{|l|}{ Gravidez } \\
\hline Sim & $16(22,5)$ \\
\hline Não & $55(77,5)$ \\
\hline \multicolumn{2}{|l|}{ Método de fertilização } \\
\hline Fertilização natural & $16(100)$ \\
\hline \multicolumn{2}{|l|}{ Filhos vivos } \\
\hline Sim & $13(81)$ \\
\hline Não & $3(19)$ \\
\hline \multicolumn{2}{|l|}{ Quantidade de filhos } \\
\hline Um & $8(62)$ \\
\hline Dois & 2(15) \\
\hline Três & $3(23)$ \\
\hline \multicolumn{2}{|l|}{ Filhos adotados } \\
\hline Sim & $1(8)$ \\
\hline Não & $12(92)$ \\
\hline
\end{tabular}

Do total de participantes, $90 \%$ referiram não estar em acompanhamento psicológico e dos que o faziam (10\%), 86\% realizam com uma frequência de uma vez por semana. Dos pacientes entre 16 e 19 anos, 37,5\% apresentavam diagnóstico nutricional de sobrepeso. Pacientes acima de 19 anos, foram diagnosticados com sobrepeso em $25 \%$ dos casos e em $13 \%$ com obesidade grau I (Tabela 4).

Referente ao uso de tabaco, $38 \%$ dos participantes já experimentaram pelo menos uma vez. $\mathrm{O}$ consumo habitual e diário está descrito na tabela 5. A faixa etária da primeira vez do uso teve média de 16 anos com desvio padrão de 3. Quanto ao uso de álcool, $84,5 \%$ dos pacientes relataram já terem experimentado algum tipo bebida alcoólica no decorrer da vida e 65\% mantinham esse consumo como hábito. A maioria $(46 \%)$ relatou uso da bebida pelo menos 1 vez por semana (Tabela 5). A idade da primeira vez do uso de 
Tabela 4. Distribuição de dados da classificação do Índice de Massa Corporal (IMC) de uma amostra de pacientes em follow up tratados em um hospital oncológico do interior paulista

\begin{tabular}{lc}
\hline Variáveis & $\mathrm{n}(\%)$ \\
\hline IMC - De 16 e 19 anos & \\
Eutrofia & $5(62,5)$ \\
Sobrepeso & $3(37,5)$ \\
IMC - Acima de 19 anos & \\
Baixo peso & $7(11)$ \\
Eutrofia & $30(48)$ \\
Sobrepeso & $16(25)$ \\
Obesidade grau I & $8(13)$ \\
Obesidade grau II & $1(1,6)$ \\
Obesidade grau III & $1(1,6)$ \\
\hline
\end{tabular}

bebida alcoólica teve média de 16 anos com desvio padrão de 3. Experimentar alguma droga ilícita foi relatada por $20 \%$ dos participantes e $7 \%$ referiram ainda fazer uso no período da pesquisa (Tabela 5). A idade da primeira vez do uso de drogas ilícitas teve média de 17 anos com desvio padrão de 2 . O tipo de droga e a frequência de uso estão relatados na tabela 5.

Tabela 5. Distribuição de dados sobre o uso de drogas lícitas e ilícitas de uma amostra de pacientes em follow up tratados em um hospital oncológico do interior paulista

\begin{tabular}{|c|c|}
\hline Variáveis & $\mathrm{n}(\%)$ \\
\hline \multicolumn{2}{|l|}{ Uso de tabaco } \\
\hline Sim & $27(38)$ \\
\hline Não & $44(62)$ \\
\hline \multicolumn{2}{|l|}{ Consumo de tabaco atual } \\
\hline Sim & $5(18,5)$ \\
\hline Não & $22(81,5)$ \\
\hline \multicolumn{2}{|l|}{ Frequência de uso (cigarros por dia) } \\
\hline 1 a 10 & $4(80)$ \\
\hline 11 a 20 & $1(20)$ \\
\hline \multicolumn{2}{|l|}{ Uso de bebida alcoólica } \\
\hline Sim & $60(84,5)$ \\
\hline Não & $11(15,5)$ \\
\hline \multicolumn{2}{|l|}{ Consumo de bebida alcoólica atual } \\
\hline $\operatorname{Sim}$ & $39(65)$ \\
\hline Não & $21(35)$ \\
\hline \multicolumn{2}{|l|}{ Frequência de uso (por semana) } \\
\hline $1 \mathrm{vez}$ & $18(46)$ \\
\hline De 2 a 3 vezes & $5(13)$ \\
\hline De 4 a 5 vezes & $1(3)$ \\
\hline Outros & $15(38)$ \\
\hline \multicolumn{2}{|l|}{ Uso de drogas ilícitas } \\
\hline Sim & $14(20)$ \\
\hline Não & $57(80)$ \\
\hline \multicolumn{2}{|l|}{ Consumo de drogas ilícitas atual } \\
\hline $\operatorname{Sim}$ & $1(7)$ \\
\hline Não & 13(93) \\
\hline \multicolumn{2}{|l|}{ Tipo de droga } \\
\hline Maconha e Dietilamida do Ácido Lisérgico (LSD) & $1(100)$ \\
\hline \multicolumn{2}{|l|}{ Frequência de uso } \\
\hline Em festas & $1(100)$ \\
\hline
\end{tabular}

\section{Discussão}

Sobreviver por cinco anos tem sido apenas o início e não o ponto final do sucesso do tratamento oncológico, uma vez que os efeitos relacionados à doença e ao tratamento causam impacto no cotidiano dos sobreviventes. ${ }^{(12)}$

Concluir o tratamento oncológico faz com que os pacientes geralmente se sintam vulneráveis, física e psicologicamente. Tornam-se intranquilos, pois temem a probabilidade de recidiva da doença e do risco de desenvolver uma segunda neoplasia; hipervigiam sua saúde pela preocupação com a aquisição de outras doenças; e são inseguros, pois se afastam do hospital e ficam distantes da equipe, que estiveram presente durante todo o tratamento e que, de certa forma, lhes transmitiam segurança. Além disso, podem desenvolver síndromes, como a do pânico e ansiedade generalizada. ${ }^{(13)}$

Trabalhar, casar e deixar de morar com os pais são os momentos de mudança nos quais os sobreviventes podem se sentir mais desamparados. Ter tido câncer e ter sido submetido a diversas modalidades terapêuticas disponíveis não deveria interferir no comportamento geral dos pacientes em serem independentes socialmente. Entretanto, alguns tumores ósseos ou do Sistema Nervoso Central (SNC), por exemplo, deixam sequelas que podem dificultar a saída de casa e a integração social. A ajuda do profissional em Psicologia pode ajudar no enfrentamento dessa situação e facilitar sua emancipação. ${ }^{(13)}$

As consequências psicológicas do câncer na infância e adolescência sugerem que os sobreviventes enfrentem danos que impactam diretamente a qualidade de vida. Outras, indicam que os sobreviventes, algumas vezes se encontram "muito bem" e por vezes melhor, do que a população sem histórico de câncer, tornando-se otimistas e com vontade de mostrar ao mundo o quanto é importante viver e ter saúde.(13,14)

O sucesso das novas modalidades terapêuticas em oncologia juntamente com a incidência de tumores em adultos jovens que estão em idade reprodutiva, repercutem também sobre a fertilidade e a vida sexual. Estima-se que a cada ano, quatro milhões de jovens brasileiros tornam-se sexualmente ativos e a tendência atual é de iniciação sexual cada vez mais cedo, principalmente nos países em desenvolvimento, como é o caso do Brasil, onde a sexarca está ocorrendo em ado- 
lescentes com 13 anos ou menos. ${ }^{(15,16)}$ Nesta pesquisa a idade média foi de 17 anos.

Quando adolescentes não possuem diálogo com os pais ou orientações relacionadas à sexualidade, se tornam vulneráveis às consequências de uma atividade sexual desprotegida. ${ }^{(15)}$

A Pesquisa Nacional de Saúde do Escolar (PeN$\mathrm{SE})^{(17)}$ avaliou o comportamento sexual de adolescentes do nono ano do ensino fundamental brasileiro e evidenciou, no ano de 2015, que cerca de $25 \%$ deles já tiveram relação sexual alguma vez e cerca de 35\% não utilizaram proteção na última relação. Nos países com maior nível educacional, a iniciação sexual ocorre mais tarde. Entre os adolescentes americanos, a sexarca é rara antes dos 12 anos de idade, ocorre em cerca de $30 \%$ entre 15 e 16 anos. ${ }^{(16)}$

É importante o conhecimento sobre sexualidade e reprodução pelos diversos profissionais que trabalham com essa população, para que antes, durante e após tratamento, os pacientes recebam informações acerca dessa temática.

Outro aspecto de grande relevância para sobreviventes é a obesidade, que pode ser decorrente de fatores relacionados ao próprio tratamento, como o uso de altas doses de corticosteroides, como também associado ao uso concomitante ou não de radioterapia craniana, podendo ocasionar problemas hormonais. Estudos apontam que escolhas alimentares pouco saudáveis, excesso de tempo gasto em atividades sedentárias e redução da atividade física são fatores que contribuem para o ganho de peso no paciente fora de terapia. ${ }^{(18)}$ Nesta pesquisa, 37,5\% dos pacientes entre 16 e 19 anos tiveram o diagnóstico nutricional de sobrepeso e cerca de $12,7 \%$ dos participantes com idades acima de 19 anos foram diagnosticados com obesidade grau I.

O uso de drogas lícitas e ilícitas são comportamentos de risco que se iniciam, geralmente, em idades precoces e se estendem por toda a vida. ${ }^{(17)} \mathrm{O}$ uso frequente dessas substâncias causam prejuízos sociais, psíquicos e biológicos, além de levar a implicações no decorrer de toda a vida. A adolescência é a faixa etária mais vulnerável para experimentação de drogas e essa, um fator preditor para o abuso de substâncias, problemas de saúde, insucesso escolar e aumento do uso de serviços de saúde. ${ }^{(17)}$ São muito os motivos que levam a esse comportamento, mas alguns fatores relacionados a essa fase da vida podem estar envolvidos, como a busca por novas experiências e o desafio à estrutura familiar e social.

Dados de 2015 mostram que 55,5\% dos adolescentes já experimentaram algum tipo de bebida alcoólica, $18,4 \%$ já utilizaram ao menos uma vez tabaco e $9 \%$ usaram drogas ilícitas. ${ }^{(17)}$ Nesta pesquisa, $84,5 \%$ dos participantes já provaram bebida alcoólica, $38 \%$ já experimentaram tabaco e 19,7\% já utilizaram, ao menos uma vez, drogas ilícitas.

Levando em consideração os aspectos descritos acima e a fase peculiar da adolescência, é importante que novas pesquisas sejam direcionadas na busca de conhecimento sobre esta população específica, uma vez que os dados evidenciados na literatura não distinguem a população oncológica da não oncológica em seus resultados de comportamentos de risco. As autoras buscaram evidenciar que alguns comportamentos de risco estão presentes na população adolescente pós tratamento e é de fundamental importância que o seguimento deste paciente ultrapasse o campo da fisiologia e patologia e seja também direcionados para os aspectos sociais e comportamentais.

Este estudo teve como limitação ter sido realizado em apenas um centro de tratamento. Estudos multicêntricos podem proporcionar uma maior representatividade da população de follow up's.

\section{Conclusão}

Os comportamentos de risco evidentes na população em geral também são observados na população de sobreviventes do câncer infantojuvenil. Estes dados podem proporcionar uma visão ampliada dos aspectos sociais demandados pelos pacientes após anos de término de terapia e auxiliar toda a equipe na prestação de cuidados para o reestabelecimento da vida desses indivíduos. $\mathrm{Na}$ prática dos profissionais que lidam com esses pacientes ainda há uma lacuna a ser preenchida com relação a essas pesquisas. Sugere-se que novos estudos no âmbito multiprofissional sejam realizados com essa população.

\section{Colaborações}

Vinhando N e Campos DR declaram que contribuíram com a concepção do estudo, análise e interpretação 
dos dados, redação do artigo, revisão crítica relevante do conteúdo intelectual e aprovação da versão final a ser publicada.

\section{Referências}

1. Instituto Nacional de Câncer José Alencar Gomes da Silva (INCA). Estimativa 2020: incidência de câncer no Brasil. Rio de Janeiro (RJ): Instituto Nacional do Câncer; 2019.

2. American Cancer Society. Risk factors and causes of childhood cancer [Internet]. Atlanta: American Cancer Society; c2019 [citado 2021 jul 12]. Disponivel em: https://www.cancer. org/cancer/cancerin-children/risk-factors-and-causes.html

3. Ministério da Saúde (BR). Protocolo de diagnóstico precoce para oncologia pediátrica. Brasília (DF): Ministério da Saúde; 2017.

4. American Cancer Society. Treating children with cancer [Internet]. Atlanta: American Cancer Society; c2019 [citado 2021 jul 12]. Disponível em: http://www.cancer.org/cancer/ cancer-in-children/how-are-childhood-cancers-treated.html

5. Dixon SB, Bjornard KL, Alberts NM, Armstrong GT, Brinkman TM, Chemaitilly W, et al. Factors influencing risk-based care of the childhood cancer survivor in the 21st century. CA Cancer J Clin. 2018;68(2):133-152.

6. Instituto Nacional do Câncer (INCA). Sobrevida de pacientes infantojuvenis com câncer é de 64\% no Brasil [internet]. Rio de Janeiro (RJ): Instituto Nacional do Câncer; 2016 [citado 2021 jul 12]. Disponível em: https://www.inca.gov.br/en/node/297

7. Hospital de Câncer de Barretos. Núcleo de Apoio ao Pesquisador. Sobrevida global 2000 à 2017. Barretos: Hospital de Câncer de Barretos; 2019.
8. Silva CP, Santos AT, Silva RP, Andrade, JD, Almeida LM. Significado dos cuidados paliativos para a qualidade da sobrevivência do paciente oncológico. Rev Bras Cancerol. 2016;3(62):225-35

9. Anders JC, Souza AIJ. Crianças e adolescentes sobreviventes ao câncer: desafios e possibilidades. Cienc Cuid Saude. 2009;8(1):31-7.

10. Teixeira JF, Maia-Lemos Pd, Cypriano MD, Pisani LP. The influence of antineoplastic treatment on the weight of survivors of childhood cancer. J Pediatr. 2016;92 (6):559-66.

11. AsfarT, Dietz NA,Arheart KL, Tannenbaum SL, McClure LA, Fleming LE, et al. Smoking behavior among adult childhood cancer survivors: what are we missing?. J Cancer Sunviv. 2016;10:131-41.

12. Oliveira BA, Lins MM, Pedrosa F, Cabral PC, Barbosa JM. Estado nutricional de crianças e adolescentes sobreviventes de leucemia linfoide aguda tratados em um centro de referência da região nordeste do Brasil. Rev Nutr. 2013;26(3):271-81.

13. Freitas JA, Oliveira BL.Aspectos psicológicos envolvidos na sobrevivência do câncer infantil. UNINGÁ Rev. 2018;55(2):1-13.

14. Turner-Sack AM, Menna R, Setchell SR. Posttraumatic growth, coping strategies, and psychological distress in adolescent survivors of cancer. J Pediatr Oncol Nurs. 2012;29(2):70-9

15. Rangel EM, Pinho LG. Interfaces no processo da educação em saúde: orientação sexual para adolescentes na escola. Linkscienceplace Interdisciplinary Scientific Journal. 2019;6(2):33-47.

16. Lara LA, Abdo CH. Aspectos da atividade sexual precoce. Rev Bras Ginecol Obstet. 2015;37(5):199-202.

17. Instituto Brasileiro de Geografia e Estatística (IBGE). Pesquisa nacional de saúde do escolar. Rio de Janeiro (RJ): Instituto Brasileiro de Geografia e Estatística; 2016.

18. Oliveira BA, Lins MM, Pedrosa F, Cabral PC, Barbosa JM. Estado nutricional de crianças e adolescentes sobreviventes de leucemia linfoide aguda tratados em um centro de referência da região nordeste do Brasil. Rev Nutr. 2013;26(3):271-81. 